\title{
Mortality Risk in People with Type 2 Diabetes: A Large Prospective Population- Based Cohort Study (The Ayrshire Diabetes Follow-Up Cohort (ADOC) Study)
}

\author{
${ }^{1}$ Diabetes Day Centre, NHS Ayrshire and Arran, University Hospital Ayr, UK \\ ${ }^{2}$ Statistics Consultant, NHS Ayrshire and Arran, University Hospital Ayr, UK \\ ${ }^{3}$ Primary Care Quality and Development, NHS Ayrshire and Arran, Ailsa Hospital, UK \\ ${ }^{4} \mathrm{AMRI}$ Institute of Diabetes and Hormonal Disorders, Kolkata, India \\ ${ }^{5}$ Glasgow Royal Infirmary, Castle St, Glasgow, UK \\ ${ }^{6}$ Institute of Cardiovascular and Medical Sciences, University of Glasgow, Glasgow, UK \\ ${ }^{7}$ Health Sciences, Division of Health Sciences, Warwick Medical School, Coventry, UK
}

Andrew Collier ${ }^{1, *}$, Mario Hair ${ }^{2}$, Lyall Cameron ${ }^{3}$, Sujoy Ghosh ${ }^{4}$, James Boyle ${ }^{5}$, Matthew Walters ${ }^{6}$ and Norman Waugh ${ }^{7}$

*Corresponding author: Andrew Collier, Diabetes Day Centre, NHS Ayrshire and Arran, University Hospital Ayr, Dalmellington Road, Ayr, UK, Tel: (44)1292 610555; E-mail: Andrew.collier@aaaht.scot.nhs.uk

Received: 22 Feb, 2021 | Accepted: 24 Mar, 2021 | Published: 31 Mar, 2021

Citation: Collier A, Hair M, Cameron L, Ghosh S, Boyle J, et al. (2021) Mortality Risk in People with Type 2 Diabetes: A Large Prospective Population-Based Cohort Study (The Ayrshire Diabetes Follow-Up Cohort (ADOC) Study). J Epidemiol Public Health Rev 6(1): dx.doi. org/10.16966/2471-8211.209

Copyright: (C) 2021 Collier A, et al. This is an open-access article distributed under the terms of the Creative Commons Attribution License, which permits unrestricted use, distribution, and reproduction in any medium, provided the original author and source are credited.

\section{Highlights}

- Ranked in order of Hazard Ratio (HR), Increasing age (HR 2.31), Smoking (HR 1.79), Ischaemic Heart Disease (IHD) (HR 1.65), Deprivation (HR 1.36), Hypertension (HR 1.23) and Male gender (HR 1.20) all increased mortality risk ( $p<0.01)$.

- This study demonstrated that a man with type 2 diabetes of 5-10 years duration who smoked, had hypertension and IHD plus lived in the most deprived area had a HR of 6.2 compared with a non-smoking, normotensive, non-diabetes subject without IHD living in the least deprived area.

- When age was adjusted for in the survival model there was an interaction between hypertension and type 2 diabetes with little increase in mortality risk. It is likely that the mechanism(s) underlying type 2 diabetes and hypertension are linked.

- Smoking prevalence decreased with duration falling from $26.8 \%$ for diabetes $<5$ years to $17.7 \%$ for diabetes $>10$ years.

- Body Mass Index $>30 \mathrm{~kg} / \mathrm{m}^{2}$ appeared to reduce mortality risk (HR $0.77, \mathrm{p}<0.01$ ).

- Abnormal lipid levels whilst not on a statin significantly increased mortality risk for raised total-cholesterol (HR 1.74) and low HDL-cholesterol (HR 1.48) but not for triglycerides (HR 0.67) (all $p<0.01$ ).

\section{Abstract}

Background: This study investigated the effects of age, gender, socio-economic status, smoking status, hypertension, ischaemic heart disease, Body Mass Index (BMI), Glycaemic control (HbA1c) and dyslipidaemia in type 2 diabetes in a population-based analysis.

Methods: Data were collected from 46 General Practice databases in 2009 and 2014. Cox regressions were run in type 2 diabetes patients plus the non-diabetes population.

Results: Individuals with type 2 diabetes $(n=16,643)$ had higher mortality rates than non-diabetes subjects. Ranked in order of Hazard Ratio (HR), increasing age (HR 2.31), smoking (HR 1.79), IHD (HR 1.65), deprivation (HR 1.36), hypertension (HR 1.23) and male gender (HR 1.20) all increased mortality risk $(p<0.01)$. Statin therapy was associated with better outcome (HR $0.65, p<0.01)$. Abnormal lipid levels whilst not on a statin significantly increased mortality risk for raised total-cholesterol (HR 1.74) and low HDL-cholesterol (HR 1.48) but not for triglycerides (HR 0.67) (all p<0.01).

Conclusions: This large study confirmed that the all-cause mortality risk in type 2 diabetes remains elevated. We demonstrated that a man with type 2 diabetes of 5-10 years duration who smoked, had hypertension and IHD plus lived in the most deprived area had a HR of 6.2 compared with a non-smoking, normotensive, non-diabetes subject without IHD living in the least deprived area. Further research is required to understand the interaction between hypertension and type 2 diabetes plus why obesity and raised triglycerides appeared to be protective in type 2 diabetes.

Keywords: Type 2 diabetes; Age; Gender; Body Mass Index (BMI); Glycaemic control; Socio-economic status; Dyslipidaemia; Hypertension; Ischaemic heart disease; Smoking status

Abbreviations: HR: Hazard Ratio; IHD: Ischaemic Heart Disease; BMI: Body Mass Index; HDL cholesterol: High-Density Lipoprotein; SIMD: Scottish Index of Multiple Deprivation; SPSS: Statistical Package for the Social Sciences; SD: Standard Deviation; UK: United Kingdom 


\section{Background}

Type 2 diabetes is a chronic complex metabolic disease and is associated with numerous macrovascular and microvascular complications [1]. The management of type 2 diabetes management is complex, expensive, involves the management of multiple risk factors, requires significant changes in lifestyle behaviors, and regular review by health care professionals [2]. The excess in mortality was considered to be primarily due to cardiovascular disease although we are becoming increasingly aware of the risk of malignancy in type 2 diabetes [3]. The evidence base for managing type 2 diabetes and preventing complications has improved greatly. Glycaemic control studies, improved cardiovascular risk factor management and smoking cessation have resulted in better outcome data and improved care [4-9].

The clinical goal of treatment in people with type 2 diabetes is to prevent the onset of associated co-morbidities and reduce the mortality risk to a level comparable to non-diabetes individuals. The aims of this study were to compare all-cause mortalities in type 2 diabetes with a non-diabetes population. We also investigated and ranked the effects of age, gender, social deprivation, body mass index (BMI), dyslipidaemia, hypertension, ischaemic heart disease and smoking status in a population-based analysis in the county of Ayrshire and Arran, Scotland over a five-year period.

\section{Methods}

Forty six out of fifty-five General Practices in NHS Ayrshire \& Arran contributed data from their practice computer systems. This covered $85 \%$ of the total patient population (above 18 years) of Ayrshire \& Arran. Data were provided in 2009 and then again in 2014. There was no significant difference in the prevalence of diabetes between practices that did and did not provide data $\left(5.5 \%\right.$ vs $5.7 \% \chi^{2}=3.3$; $\mathrm{p}=0.07$ ).

In a recently published parallel study, we investigated the outcome of patients with type 1 diabetes over the same five-year period where further information on methodology is available [10]. In both studies, survival was measured in days from a 'start date' of 1/10/2009 to an 'end date' of 30/09/2014. The number of days between the start and end date (survival time) was calculated for each subject. Data on age, gender, deprivation [11], smoking status, hypertension and ischaemic heart disease, were collected for all subjects. Ischaemic heart disease was defined as per the Quality Outcome Framework document $[12,13]$. Data on glycaemic control and lipid levels were only collected for those patients with diabetes.

Ethical opinion was not required for this study. The audit was registered with the Clinical Governance Department, NHS Ayrshire and Arran, UK and Caldicott Guardian approval was obtained from each General Practice.

\section{Statistics}

Statistical analysis mirrored that of our recent study [10]. A Cox regression was run on survival time with type 2 diabetes, gender, age (in decades), deprivation (quintile), smoking, hypertension and IHD as covariates. Interactions of diabetes groups with all the other covariates were also included. Reference group were female, quintile 1 (least deprived), non-diabetes, non-smoker, normotensive and no IHD [10].

BMI data was not included in the initial survival model. Instead a matched sample of two non-diabetes subjects for each type 2 diabetes subject was selected using propensity score matched on the latest BMI reading. After matching there was data on 49371 subjects (66.6\% nondiabetes subjects and $33.3 \%$ with type 2 diabetes) and the two groups had similar BMI (type 2 diabetes $31.21 \pm 6.88$ (Mean \pm SD) vs nondiabetes $31.17 \pm 6.84, \mathrm{p}=0.52$ ). The previous Cox regression was run on this subset with BMI $>30 \mathrm{k} / \mathrm{m}^{2}$ included as a covariate.

A Cox regression including only patients with type 2 diabetes was also run using the same covariates as previously but also included BMI, glycaemic control, lipid levels and complications. Baseline categories were as above; the baseline for diabetes was duration of less than 5 years. Statistical analysis was performed using SPSS V21.

\section{Results}

In total 269,947 subjects were tracked from 2009 to 2014 . $93.8 \%$ were non-diabetes subjects. $6.2 \%$ (16643) had type 2 diabetes. $2.3 \%$ (6260) had diabetes for less than 5 years, 2\% (5353) had diabetes for between 5 and 10 years and 1.9\% (5030) had diabetes for more than 10 years. Between 2009 and 2014, 4.5\% of the subjects died and $6.5 \%$ moved from the practices included in the study; the remaining $89.1 \%$ remained registered. All-cause mortality was 3.9\% for non-diabetes subjects but was significantly higher for type 2 diabetes. Mortality was lowest for duration of diabetes less than 5 years and greatest for those with duration $5-10$ years ( $<5$ years $10.1 \%$, OR 2.8 ; 5-10 years $14.7 \%$, OR $4.2 ;>10$ years $13.9 \%$, OR 4.0 ) (Table 1 ).

\section{Gender and age}

There were significantly more males among the patients with type 2 diabetes (regardless of duration) than non-diabetes subjects $(55 \%$ vs $49 \%)\left(\chi^{2}=254.7, \mathrm{p}<0.01\right)$. Non-diabetes subjects were significantly younger than type 2 diabetes groups ( (Mean \pm SD) $45.2 \pm 22.2$ vs 64.3 \pm 13.2 (diabetes $<5$ years) vs $68.1 \pm 12.2$ ( 5 - 10 years) and $71.1 \pm 11.1$ ( $>10$ years), all $\mathrm{p}<0.01)$. Females were older than males for all groups [mean diff (95\% CI) non-diabetes 3.0 years $(2.8,3.2)$; diabetes $<5$ years 1.8 years $(1.2,2.5)$; diabetes 5 - 10 years: 2.0 years $(1.4,2.6)$; diabetes $>10$ years: 2.0 years $(1.4,2.6)$ : all $\mathrm{p}<0.01]$.

Relative mortality of patients with type 2 diabetes compared to non-diabetes subjects was similar for both males and females (Table 1). Once age was taken into account, the survival analysis (Table 2), showed that mortality risk was higher for males. There was no gender difference or interaction by duration of diabetes. Mortality increased with age and was higher for type 2 diabetes across all age groups.

\section{Deprivation and smoking}

Deprivation was Health Board weighted [9,12]. For type 2diabetes (regardless of duration) there was a clear pattern. Deprivation increased from $15.1 \%$ in the least deprived quintile to $23.5 \%$ in the most deprived $\left(\mathrm{X}^{2}=236.3, \mathrm{p}<0.001\right)$ (Figure 1). Mortality increased with increasing deprivation and was consistently higher for type 2diabetes in each quintile (Figure 1). The mortality differential between patients with type 2 diabetes and non-diabetes subjects remained similar and in line with the overall differential found earlier (Table 1). Both deprivation and type 2 diabetes were associated with increased mortality. There was, however, no evidence of an interaction effect.

Smoking prevalence at baseline was $22 \%$ for non-diabetes subjects. For type 2 diabetes prevalence decreased with duration falling from $26.8 \%$ for diabetes $<5$ years; $21.3 \%$ for diabetes $5-10$ years to $17.7 \%$ for diabetes $>10$ years. Mortality rates were higher for smokers than non-smokers, but the differential was smaller among patients with type 2 diabetes. Among non-smokers mortality was around four times higher for patients with type 2 diabetes (depending on duration) while among smokers' mortality was only around three times higher 
Table 1: Mortality by diabetes duration (not adjusted for covariates).

\begin{tabular}{|c|c|c|c|c|c|c|c|}
\hline & \multirow{2}{*}{$\begin{array}{l}\text { Non diabetes } \\
n=253304\end{array}$} & \multicolumn{3}{|c|}{ Type 2 diabetes } & \multicolumn{3}{|c|}{ OR $(95 \% \mathrm{Cl})$ Mortality vs Non-diabetes } \\
\hline & & $\begin{array}{c}<5 \text { yrs } \\
n=6260\end{array}$ & $\begin{array}{l}5-10 \text { yrs } \\
n=5353\end{array}$ & $\begin{array}{l}>10 \text { yrs } \\
n=5030\end{array}$ & $<5$ yrs & $5-10$ yrs & $>10$ yrs \\
\hline Total & $3.9 \%(9923)$ & $10.1 \%(632)$ & $14.7 \%(785)$ & $13.9 \%(698)$ & $2.8(2.5,3.0)$ & $4.2(3.9,4.6)$ & $4.0(3.6,4.3)$ \\
\hline \multicolumn{8}{|l|}{ Gender } \\
\hline Female & $4.1 \%(5370)$ & $10.2 \%(289)$ & $14.6 \%(353)$ & $14.1 \%(319)$ & $2.6(2.3,3.0)$ & $4.0(3.5,4.5)$ & $3.8(3.4,4.3)$ \\
\hline Male & $3.7 \%(4553)$ & $10.0 \%(343)$ & $14.7 \%(432)$ & $13.7 \%(379)$ & $2.9(2.6,3.2)$ & $4.5(4.0,5.0)$ & $4.1(3.7,4.6)$ \\
\hline \multicolumn{8}{|l|}{ Age* } \\
\hline$<49$ & $0.4 \%(531)$ & $2.3 \%(20)$ & $2.0 \%(8)$ & $3.3 \%(6)$ & $6.3(4.0,9.9)$ & $5.4(2.7,10.9)$ & $9.2(4.1,20.9)$ \\
\hline $50-59$ & $1.6 \%(630)$ & $3.8 \%(52)$ & $3.8 \%(34)$ & $3.1 \%(20)$ & $2.4(1.8,3.1)$ & $2.3(1.6,3.3)$ & $1.9(1.2,3.0)$ \\
\hline $60-69$ & $4.4 \%(1436)$ & $7.1 \%(122)$ & $9.9 \%(147)$ & $6.5 \%(82)$ & $1.7(1.4,2.0)$ & $2.4(2.0,2.8)$ & $1.5(1.2,1.9)$ \\
\hline $70-79$ & $11.1 \%(2532)$ & $13.6 \%(203)$ & $18.1 \%(286)$ & $15.2 \%(265)$ & $1.3(1.1,1.5)$ & $1.8(1.6,2.0)$ & $1.4(1.3,1.7)$ \\
\hline $80+$ & $28.4 \%(4793)$ & $29.6 \%(235)$ & $31.7 \%(310)$ & $27.1 \%(325)$ & $1.1 \mathrm{NS} p=.47$ & $1.2(1.0,1.30)$ & 0.9 NS $p=.35$ \\
\hline \multicolumn{8}{|l|}{ Deprivation* } \\
\hline Quintile 1 & $3.7 \%(1727)$ & $10.5 \%(94)$ & $13.3 \%(110)$ & $12.9 \%(99)$ & $3.1(2.5,3.8)$ & $4.0(3.3,4.9)$ & $3.9(3.1,4.8)$ \\
\hline Quintile 2 & $2.8 \%$ (1399) & $7.8 \%(88)$ & $10.9 \%(104)$ & $9.2 \%(77)$ & $3.0(2.4,3.7)$ & $4.3(3.5,5.3)$ & $3.5(2.8,4.5)$ \\
\hline Quintile 3 & $4.3 \%(2157)$ & $11.2 \%(143)$ & $15.2 \%(168)$ & $14.2 \%(154)$ & $2.8(2.3,3.3)$ & $4.0(3.3,4.70$ & $3.7(3.1,4.4)$ \\
\hline Quintile 4 & $4.6 \%(2376)$ & $11.6 \%(165)$ & $16.4 \%(191)$ & $15.8 \%(185)$ & $2.7(2.3,3.2)$ & $4.1(3.5,4.8)$ & $3.9(3.3,4.6)$ \\
\hline Quintile 5 & $4.1 \%(2123)$ & $9.0 \%(134)$ & $15.6 \%(196)$ & $15.2 \%(173)$ & $2.3(1.9,2.8)$ & $4.3(3.7,5.1)$ & $4.2(3.6,5.0)$ \\
\hline \multicolumn{8}{|l|}{ Smoking* } \\
\hline Non smoker & $3.7 \%(7095)$ & $10.1 \%(461)$ & $14.5 \%(611)$ & $13.7 \%(568)$ & $2.9(2.6,3.2)$ & $4.4(4.0,4.8)$ & $4.1(3.7,4.5)$ \\
\hline Smoker & $5.0 \%(2687)$ & $10.3 \%(169)$ & $15.3 \%(174)$ & $14.6 \%(130)$ & $2.2(1.8,2.5)$ & $3.4(2.9,4.0)$ & $3.2(2.7,3.9)$ \\
\hline \multicolumn{8}{|l|}{ Hypertension } \\
\hline Normotensive & $2.6 \%(5939)$ & $7.9 \%(310)$ & $11.9 \%(280)$ & $12.0 \%(224)$ & $3.2(2.8,3.6)$ & $5.0(4.4,5.6)$ & $5.0(4.4,5.8)$ \\
\hline Throughout & $14.0 \%$ (3984) & $13.7 \%(322)$ & $16.8 \%(505)$ & $15.0 \%(474)$ & $1.0 \mathrm{NS} p=.75$ & $1.2(1.1,1.4)$ & $1.1 \mathrm{NS} p=.14$ \\
\hline \multicolumn{8}{|l|}{ IHD } \\
\hline No & $3.1 \%(7644)$ & $7.8 \%(415)$ & $11.5 \%(481)$ & $10.8 \%(395)$ & $2.6(2.4,2.9)$ & $4.0(3.6,4.4)$ & $3.7(3.3,4.1)$ \\
\hline Throughout & $23.0 \%$ (2279) & $22.5 \%(217)$ & $26.1 \%(304)$ & $22.3 \%(303)$ & $1.0 \mathrm{NS} p=.71$ & $1.2(1.0,1.4)$ & $1.0 \mathrm{NS} p=.58$ \\
\hline
\end{tabular}

*Missing values: Smoking 13496 (5.0\%) Deprivation 2234 (0.9\%) Age 25 (0.009\%).

Table 2: Regression coefficients and effect sizes for factors in the cox regression model comparing type 2 diabetes and non-diabetes subjects (adjusted for covariates).

\begin{tabular}{|c|c|c|c|}
\hline Factor & Beta \pm SE & $\mathbf{p}$ & HR (95\% CI) \\
\hline \multicolumn{4}{|l|}{ Type 2 DM compared to non-diabetics } \\
\hline Diabetes duration $<5$ yrs & $0.22 \pm 0.06$ & $<0.01$ & $1.24(1.11,1.39)$ \\
\hline Diabetes duration 5-10 yrs & $0.36 \pm 0.06$ & $<0.01$ & $1.43(1.27,1.62)$ \\
\hline Diabetes duration $>10$ yrs & $0.19 \pm 0.07$ & $<0.01$ & $1.21(1.06,1.39)$ \\
\hline Age (decades) & $0.84 \pm 0.01$ & $<0.01$ & $2.31(2.28,2.35)$ \\
\hline Gender (Male) & $0.18 \pm 0.02$ & $<0.01$ & $1.20(1.15,1.25)$ \\
\hline \multicolumn{4}{|l|}{ Deprivation compared to Q1 } \\
\hline Quintile 2 & $-0.12 \pm 0.03$ & $<0.01$ & $0.89(0.83,0.95)$ \\
\hline Quintile 3 & $0.20 \pm 0.03$ & $<0.01$ & $1.22(1.15,1.30)$ \\
\hline Quintile 4 & $0.31 \pm 0.03$ & $<0.01$ & $1.36(1.29,1.45)$ \\
\hline Quintile 5 & $0.31 \pm 0.03$ & $<0.01$ & $1.36(1.29,1.45)$ \\
\hline Smoker compared to non-smoker & $0.58 \pm 0.02$ & $<0.01$ & $1.79(1.71,1.87)$ \\
\hline IHD compared to none & $0.50 \pm 0.02$ & $<0.01$ & $1.65(1.59,1.75)$ \\
\hline Hypertensive compared to normotensive & $0.21 \pm 0.02$ & $<0.01$ & $1.23(1.18,1.28)$ \\
\hline \multicolumn{4}{|l|}{ Diabetes group *Hypertension } \\
\hline Diabetes duration $<5$ yrs and hypertension & $-0.07 \pm 0.08$ & 0.39 & 0.93 NS \\
\hline Diabetes duration 5-10 yrs and hypertension & $-0.13 \pm 0.08$ & 0.10 & $0.88 \mathrm{NS}$ \\
\hline Diabetes duration $>10$ yrs and hypertension & $-0.24 \pm 0.08$ & $<0.01$ & $0.79(0.67,0.93)$ \\
\hline
\end{tabular}

Final model Cox and Snell R of $0.10(p<0.01)$.

*Missing cases $4.4 \%$ A Cox regression that omitted smoking was undertaken with far fewer missing cases (0.9\%). This analysis showed no substantial changes for all the other covariates.

Citation: Collier A, Hair M, Cameron L, Ghosh S, Boyle J, et al. (2021) Mortality Risk in People with Type 2 Diabetes: A Large Prospective Population-Based Cohort Study (The Ayrshire Diabetes Follow-Up Cohort (ADOC) Study). J Epidemiol Public Health Rev 6(1): dx.doi. 
Table 3: Mortality by BMI in non-diabetic subjects and type 2 diabetes according to duration (matched file).

\begin{tabular}{|c|c|c|c|c|c|c|}
\hline \multirow[b]{2}{*}{ Type of patient } & \multirow[b]{2}{*}{$\mathbf{n}$} & \multirow{2}{*}{$\begin{array}{l}\% \text { with BMI } \\
\left(\geq 30 \mathrm{~kg} / \mathrm{m}^{2}\right)\end{array}$} & \multicolumn{4}{|c|}{ Mortality } \\
\hline & & & Overall & BMI $\left(<30 \mathrm{~kg} / \mathrm{m}^{2}\right)$ & Raised BMI $\left(\geq 30 \mathrm{~kg} / \mathrm{m}^{2}\right)$ & $\begin{array}{c}\text { OR }(95 \% \mathrm{Cl}) \mathrm{BMI} \geq 30 \mathrm{~kg} / \mathrm{m}^{2} \text { vs }<30 \\
\mathrm{~kg} / \mathrm{m}^{2}\end{array}$ \\
\hline Non-diabetes & 32907 & $52.4 \%(17238)$ & $3.9 \%(1274)$ & $4.8 \%(752)$ & $3.0 \%(522)$ & $0.62(0.55,0.69) p<0.001$ \\
\hline $\begin{array}{l}\text { Diabetes duration } \\
<5 \text { yrs }\end{array}$ & 6150 & $56.6 \%(3481)$ & $9.6 \%(593)$ & $14.3 \%(382)$ & $6.1 \%(211)$ & $0.39(0.32,0.46) p<0.001$ \\
\hline $\begin{array}{l}\text { Diabetes duration } \\
\text { 5-10 yrs }\end{array}$ & 5324 & $50.9 \%(2712)$ & $14.5 \%(772)$ & $18.9 \%(494)$ & $10.3 \%(278)$ & $0.49(0.42,0.57) p<0.001$ \\
\hline $\begin{array}{l}\text { Diabetes duration } \\
>10 \text { yrs }\end{array}$ & 4990 & $49.3 \%(2462)$ & $13.7 \%(685)$ & $17.6 \%(444)$ & $9.8 \%(241)$ & $0.51(0.43,0.60) p<0.001$ \\
\hline Total & 49371 & $52.4 \%(25893)$ & $6.7 \%(3324)$ & $8.8 \%(2072)$ & $4.8 \%(1252)$ & $0.53(0.49,0.57) p<0.001$ \\
\hline
\end{tabular}

Table 4: Mortality in type 2 diabetes by lipids levels and complications (not adjusted for covariates).

\begin{tabular}{|c|c|c|c|c|c|c|}
\hline & \multicolumn{6}{|c|}{ Duration of diabetes } \\
\hline & \multicolumn{2}{|c|}{$<5$ yrs $(n=6260)$} & \multicolumn{2}{|c|}{$5-10$ yrs $(n=5353)$} & \multicolumn{2}{|c|}{$>10$ yrs $(n=5030)$} \\
\hline Mortality & \multicolumn{2}{|c|}{$10.1 \%(632)$} & \multicolumn{2}{|c|}{$14.7 \%(795)$} & \multicolumn{2}{|c|}{$13.9 \%(698)$} \\
\hline HbA1c (Mean \pm SD) & & Diff $(95 \% \mathrm{Cl})$ & & Diff $(95 \% \mathrm{Cl})$ & & Diff $(95 \% \mathrm{Cl})$ \\
\hline Survived\% (mmol/mol) & $\begin{array}{c}7.0 \pm 1.3 \\
(52.7 \pm 14.3)\end{array}$ & \multirow{2}{*}{ NS $p=0.93$} & $\begin{array}{c}7.3 \pm 1.5 \\
(56.2 \pm 16.4)\end{array}$ & \multirow{2}{*}{$\begin{array}{c}2.64 \\
(1.39,3.89)\end{array}$} & $\begin{array}{c}7.6 \pm 1.6 \\
(60.0 \pm 17.5)\end{array}$ & \multirow{2}{*}{$\begin{array}{c}3.91 \\
(2.51,5.33)\end{array}$} \\
\hline Deceased\% (mmol/mol) & $\begin{array}{c}7.0 \pm 1.5 \\
(52.6 \pm 16.8)\end{array}$ & & $\begin{array}{c}7.1 \pm 1.6 \\
(53.6 \pm 17.3)\end{array}$ & & $\begin{array}{c}7.3 \pm 1.6 \\
(56.1 \pm 17.7)\end{array}$ & \\
\hline On statin therapy & & $\mathrm{OR}^{+}(95 \% \mathrm{Cl})$ & & $\mathrm{OR}^{+}(95 \% \mathrm{Cl})$ & & $\mathrm{OR}^{+}(95 \% \mathrm{Cl})$ \\
\hline No & $12.8 \%(263)$ & \multirow{2}{*}{$\begin{array}{c}0.66 \\
(0.55,0.76)\end{array}$} & $20.1 \%(255)$ & \multirow{2}{*}{$\begin{array}{c}0.59 \\
(0.50,0.70)\end{array}$} & $20.9 \%(215)$ & \multirow{2}{*}{$\begin{array}{c}0.52 \\
(0.44,0.62)\end{array}$} \\
\hline Yes & $8.8 \%(369)$ & & $13.0 \%(530)$ & & $12.1 \%(483)$ & \\
\hline \multicolumn{7}{|l|}{ Cholesterol } \\
\hline$<5 \mathrm{mmol} / \mathrm{l}$ & $12.1 \%(146)$ & \multirow[b]{2}{*}{$1.11 \mathrm{NS} p=0.43$} & $18.1 \%(149)$ & \multirow{2}{*}{$\begin{array}{c}1.43 \\
(1.08,1.89)\end{array}$} & $19.4 \% \%(141)$ & \multirow[b]{2}{*}{$1.30 \mathrm{NS} p=0.12$} \\
\hline$\geq 5 \mathrm{mmol} / \mathrm{l}$ and not on statin therapy & $13.3 \%(106)$ & & $24.0 \%(106)$ & & $23.8 \%(71)$ & \\
\hline \multicolumn{7}{|l|}{ HDL-cholesterol } \\
\hline $\begin{array}{l}\text { Male } \geq 1 \mathrm{mmol} / \mathrm{l} \\
\text { Female } \geq 1.3 \mathrm{mmol} / \mathrm{l}\end{array}$ & $14.2 \%(127)$ & \multirow[b]{2}{*}{$\begin{array}{c}0.65 \\
(0.49,0.86)\end{array}$} & $21.1 \%(130)$ & \multirow[b]{2}{*}{0.84 NS $p=0.22$} & $23.2 \%(130)$ & \multirow[b]{2}{*}{$\begin{array}{l}0.73 \mathrm{NS} \\
(P=0.06)\end{array}$} \\
\hline $\begin{array}{l}\text { Male }<1 \mathrm{mmol} / \mathrm{l} \\
\text { Female }<1.3 \mathrm{mmol} / \mathrm{l} \text { and not on } \\
\text { statin therapy }\end{array}$ & $9.7 \%(102)$ & & $18.3 \%(112)$ & & $18.1 \%(77)$ & \\
\hline \multicolumn{7}{|l|}{ Non-fasting triglycerides } \\
\hline$<1.7 \mathrm{mmol} / \mathrm{l}$ & $11.7 \%(133)$ & \multirow[b]{2}{*}{1.02 NS $p=0.90$} & $18.9 \%(135)$ & \multirow[b]{2}{*}{$1.11 \mathrm{NS} p=0.49$} & $16.7 \%(97)$ & \multirow{2}{*}{$\begin{array}{c}1.75 \\
(1.28,2.39)\end{array}$} \\
\hline $\begin{array}{l}\geq 1.7 \mathrm{mmol} / \mathrm{l} \text { and not on statin } \\
\text { therapy }\end{array}$ & $11.9 \%(96)$ & & $20.4 \%(103)$ & & $26.0 \%(104)$ & \\
\hline \multicolumn{7}{|l|}{ Retinopathy } \\
\hline No & $11.4 \%(457)$ & \multirow{2}{*}{$\begin{array}{c}0.66 \\
(0.55,0.79)\end{array}$} & $18.7 \%(430)$ & \multirow{2}{*}{$0.57(0.49,0.67)$} & $17.4 \%(256)$ & \multirow{2}{*}{$\begin{array}{c}0.68 \\
(0.57,0.80)\end{array}$} \\
\hline Yes & $7.8 \%(175)$ & & $11.6 \%(355)$ & & $12.4 \%(442)$ & \\
\hline \multicolumn{7}{|l|}{ Neuropathy/Foot Ulcer ${ }^{1}$} \\
\hline No & $10.0 \%(611)$ & \multirow{2}{*}{$1.92(1.19,3.10)$} & $13.9 \%(704)$ & \multirow{2}{*}{$2.27(1.74,2.97)$} & $13.3 \%(603)$ & \multirow{2}{*}{$\begin{array}{c}1.48 \\
(1.17,1.88)\end{array}$} \\
\hline Yes & $17.5 \%(21)$ & & $26.9 \%(81)$ & & $18.6 \%(95)$ & \\
\hline
\end{tabular}

Missing values: Triglycerides 361 (2.2\%), HDL 387 (2.3\%) Cholesterol 75 (0.5\%)

${ }^{1}$ The prevalence of both neuropathy and foot ulcer were low (around $5 \%$ ) and the overlap between the two conditions was small so the two conditions were merged to give more robust estimates. 
Table 5: Regression coefficients and effect sizes for factors in the cox regression model of type 2 diabetes (adjusted for covariates).

\begin{tabular}{|c|c|c|c|}
\hline Factor & Beta \pm SE & $\mathbf{p}$ & $\mathrm{HR}(95 \% \mathrm{Cl})$ \\
\hline \multicolumn{4}{|c|}{ Type 2 diabetes compared to diabetes duration $<5$ yrs } \\
\hline Diabetes duration 5-10 yrs & $0.16 \pm 0.07$ & 0.03 & $1.17(1.02,1.36)$ \\
\hline Diabetes duration $>10$ yrs & $-0.03 \pm 0.08$ & 0.72 & $0.97 \mathrm{NS}$ \\
\hline Age (decades) & $0.58 \pm 0.02$ & $<.01$ & $1.79(1.71,1.88)$ \\
\hline Gender (Male) & $0.18 \pm 0.05$ & $<.01$ & $1.19(1.08,1.31)$ \\
\hline $\mathrm{BMI} \geq 30 \mathrm{k} / \mathrm{m}^{2}$ and diabetes duration $<5 \mathrm{yrs}$ & $-0.54 \pm 0.09$ & $<.01$ & $0.58(0.48,0.70)$ \\
\hline $\mathrm{BMI} \geq 30 \mathrm{k} / \mathrm{m}^{2}$ and diabetes duration $5-10 \mathrm{yrs}$ & $-0.25 \pm 0.08$ & 0.02 & $0.78(0.66,0.91)$ \\
\hline $\mathrm{BMI} \geq 30 \mathrm{k} / \mathrm{m}^{2}$ and diabetes duration $>10 \mathrm{yrs}$ & $-0.22 \pm 0.09$ & $<.01$ & $0.81(0.68,0.95)$ \\
\hline \multicolumn{4}{|l|}{ Deprivation compared to Q1 } \\
\hline Quintile 2 & $-0.21 \pm 0.09$ & 0.02 & $0.81(0.68,0.97)$ \\
\hline Quintile 3 & $0.11 \pm 0.08$ & 0.15 & $1.12 \mathrm{NS}$ \\
\hline Quintile 4 & $0.26 \pm 0.08$ & $<.01$ & $1.30(1.12,1.51)$ \\
\hline Quintile 5 & $0.21 \pm 0.08$ & $<.01$ & $1.23(1.05,1.43)$ \\
\hline Smoker compared to non-smoker & $0.32 \pm 0.06$ & $<.01$ & $1.38(1.23,1.55)$ \\
\hline Hypertensive compared to normotensive & $0.16 \pm 0.05$ & $<.01$ & $1.17(1.06,1.29)$ \\
\hline IHD compared to none & $0.65 \pm 0.05$ & $<.01$ & $1.92(1.74,2.11)$ \\
\hline HbA1c & $0.02 \pm 0.02$ & 0.22 & $1.02 \mathrm{NS}$ \\
\hline On statin therapy & $-0.43 \pm 0.07$ & $<.01$ & $0.65(0.56,0.76)$ \\
\hline Raised cholesterol and not on statin therapy & $0.56 \pm 0.09$ & $<.01$ & $1.74(1.47,2.06)$ \\
\hline Low HDL-cholesterol and not on statin therapy & $0.39 \pm 0.09$ & $<.01$ & $1.48(1.24,1.75)$ \\
\hline Raised triglycerides and not on statin therapy & $-0.40 \pm 0.09$ & $<.01$ & $0.67(0.56,0.80)$ \\
\hline Retinopathy & $-0.36 \pm 0.05$ & $<.01$ & $0.70(0.63,0.77)$ \\
\hline Ulcer/Neuropathy & $0.43 \pm 0.08$ & $<.01$ & $1.53(1.31,1.79)$ \\
\hline
\end{tabular}

Final model Cox and Snell R of $0.12(p<0.01)$.

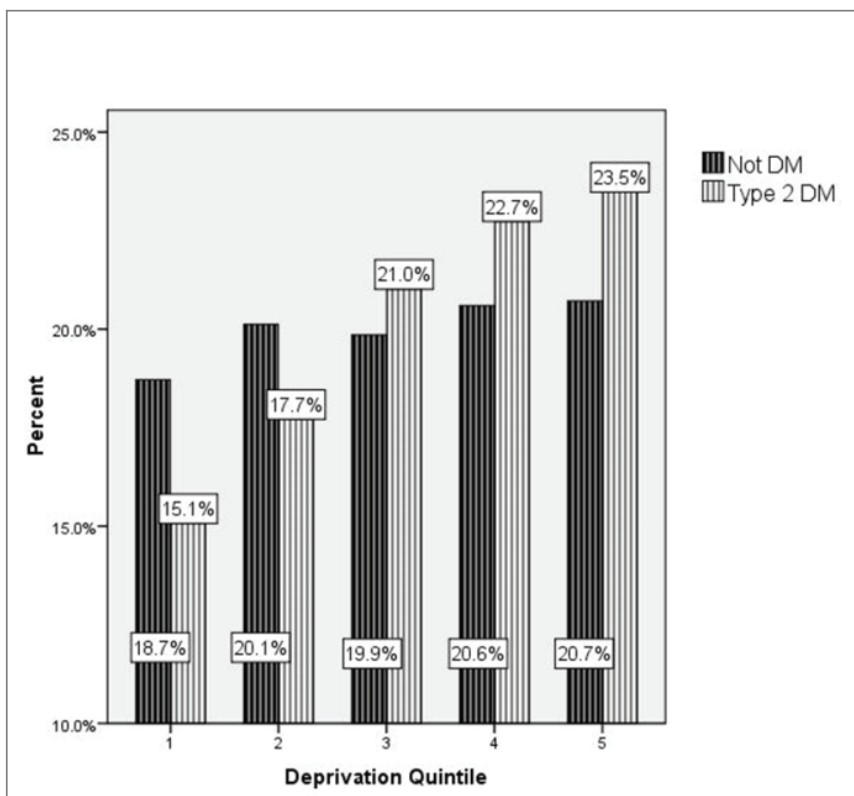

Figure 1: Deprivation for non-diabetes subjects and type 2 diabetes by quintiles. for patients with type 2 diabetes. Once other factors were considered there was no evidence of an interaction between smoking and type 2 diabetes as confirmed by the survival analysis (Table 2).

\section{Hypertension and Ischaemic Heart Disease (IHD)}

The prevalence of hypertension at baseline was $11.2 \%$ for nondiabetes. For type 2 diabetes, the prevalence of hypertension increased with duration rising from $37.4 \%$ for diabetes duration $<5$ years; $56.0 \%$ for diabetes duration $5-10$ years to $63.0 \%$ for diabetes duration $>10$ years $(\mathrm{p}<0.01)$. Mortality rates were higher for hypertensive patients compared with normotensive patients, but the differential was smaller among patients with type 2 diabetes. In individuals with hypertension, mortality was similar for both non-diabetes individuals and patients with type 2 diabetes (Table 1). Once other factors were considered there was still evidence of an interaction between hypertension and type 2 diabetes as confirmed by the survival analysis (Table 2). This suggests that the increase in mortality risk from a combination of type 2 diabetes and hypertension was not simply additive.

The prevalence of IHD at baseline was $3.9 \%$ for non-diabetes subjects. For type 2 diabetes the prevalence increased with duration rising from $15.4 \%$ for diabetes duration $<5$ years; $21.7 \%$ for diabetes duration $5-10$ years to $27.0 \%$ for diabetes duration $>10$ years $(p<0.01)$. Mortality rates were higher for IHD than non-IHD patients, but the differential was smaller among patients with type 2 diabetes. Among patients with no history of IHD, mortality risk was around three to

Citation: Collier A, Hair M, Cameron L, Ghosh S, Boyle J, et al. (2021) Mortality Risk in People with Type 2 Diabetes: A Large Prospective Population-Based Cohort Study (The Ayrshire Diabetes Follow-Up Cohort (ADOC) Study). J Epidemiol Public Health Rev 6(1): dx.doi. 
four times higher for type 2 diabetes (depending on duration). Among IHD patients, however, mortality was similar for both non-diabetes subjects and type 2 diabetes patients (Table 1). Once other factors, particularly age, were considered there was no evidence of an interaction between IHD and type 2 diabetes as confirmed by the survival analysis (Table 2).

\section{Survival analysis}

The Cox regression model which compared patients with type 2 diabetes and non-diabetes subjects included $95.6 \%$ of cases. 12,003 (4.4\%) of the whole cohort were excluded mostly due to lack of smoking data. There were 11,717 (4.3\%) deaths among the included cases. The effect size (HR) and significance (p) for the variables in the model are available in table 2. Only significant interactions are included.

When all other factors were considered, patients with type 2 diabetes had higher mortality risk than non-diabetes individuals. Increasing age, deprivation, being male, smoking, hypertension and IHD all increased mortality risk. Apart from hypertension, there were no significant interaction effects with type 2 diabetes. Patients with type 2 diabetes and hypertension had a lower mortality risk than would be expected from the individual risk factors although this was only statistically significant for those with diabetes for over 10 years (Table 1).

\section{Body mass index (matched sample)}

In the smaller matched sample, $52.4 \%$ of non-diabetes subjects and $52.6 \%$ of type 2 diabetes had a BMI at $>30 \mathrm{~kg} / \mathrm{m}^{2}(\mathrm{p}=0.70)$. The prevalence of BMI at $>30 \mathrm{~kg} / \mathrm{m}^{2}$ decreased with duration of diabetes falling from $56.6 \%$ for $<5$ years to $49.3 \%$ for $>10$ years (Table 3 ).

Mortality was significantly lower for those with BMI $>30 \mathrm{~kg} / \mathrm{m}^{2}$ for all groups (Table 3). However, those with BMI $>30 \mathrm{~kg} / \mathrm{m}^{2}$ were significantly younger than those with lower BMI in all type 2 diabetes groups ( $<5$ years: $60.9 \pm 12.6$ vs $68.5 \pm 12.4 ; 5-10$ years $64.5 \pm 11.6 v s$ $71.8 \pm 11.8 ;>10$ years: $67.9 \pm 10.5$ vs $74.1 \pm 10.8$; all $\mathrm{p}<.001)$ and this will have exaggerated the difference in mortality rates.

A similar survival model was undertaken on the matched file with the addition of BMI as a covariate. The Hazard Ratio (HR) for BMI $>30 \mathrm{~kg} / \mathrm{m}^{2}$ was 0.77 (95\% CI: $0.71,0.83$ ) indicating that those with $\mathrm{BMI}>30 \mathrm{~kg} / \mathrm{m}^{2}$ had a lower mortality risk once other covariates were considered. There was no significant interaction between diabetes and $\mathrm{BMI}$ and there were no substantive differences on any of the other covariates included in the previous model.

\section{Glycaemic control and lipid levels (type 2 diabetes patients only)}

HbAlc and lipids were only routinely measured in subjects with type 2 diabetes. The latest reading was used in the analysis. There was no significant difference in mean $\mathrm{HbAlc}$ between those who survived and those who died when diabetes duration was less than 5 years. For subjects with diabetes duration of over 5 years, those who survived had a slightly but significantly higher mean HbA1c (Table 4).

$7.5 \%$ (1249) had no defined lipids abnormality and were not on statin therapy. $73.9 \%$ (12293) were on statin therapy. The prevalence of statin therapy increased by duration of diabetes $(<5$ years $67.2 \%$; $5-10$ years $76.3 \%$; $>10$ years $79.5 \%$; $p<0.01) .18 .6 \%$ (3101) had at least one defined lipid abnormality and were not on statin therapy. The prevalence a lipid abnormality decreased with duration of diabetes ( $<5$ years $23.9 \%$; $5-10$ years $17.1 \%$; $>10$ years $13.6 \%$; $p<0.01$ ). This is consistent with a lipid abnormality being associated with increasing mortality.
9.3\% (1538) had a total-cholesterol $>5 \mathrm{mmol} / \mathrm{L}$ but were not on statin therapy; $10.5 \%$ (1714) had a low HDL-cholesterol but were not on statin therapy. $12.9 \%$ (2095) had non-fasting triglycerides $>1.7$ $\mathrm{mmol} / \mathrm{L}$ and were not statin therapy. In all cases prevalence of these abnormalities decreased with duration (total-cholesterol 12.9\%; 8.2\%; 5.9\%: HDL-cholesterol 13.3\%; 9.6\%; 8.1\%: non-fasting triglycerides $17.3 \%$; $11.7 \%$; $8.6 \%$ : all $\mathrm{p}<0.01$ ) which is again consistent with increasing mortality.

Those on statin therapy had significantly lower mortality risk regardless of duration of diabetes. Those not on statin therapy and with a raised cholesterol and low HDL-cholesterol had higher mortality than those with normal levels. Probably due to the relatively small numbers, the difference was only significant for those with 5-10 years diabetes duration (increased total-cholesterol) and over 10 years duration (reduced HDL-cholesterol). For non-fasting triglycerides, mortality was lower for those with levels $>1.7 \mathrm{mmol} / \mathrm{L}$ but was only significant for those with diabetes duration of less than 5 years (Table 4).

\section{Diabetes complications}

The prevalence of retinopathy was $53.2 \%$ while the prevalence of neuropathy/foot ulcer was $5.6 \%$. As expected, the prevalence of both increased with duration of diabetes (retinopathy $36.0 \% ; 57.0 \% ; 70.7 \%$ : neuropathy/foot ulcer $1.9 \%$; $5.6 \% ; 10.2 \%$ : all p 0.01 ). Mortality was significantly higher for those with neuropathy/foot ulcer regardless of duration of diabetes (Table 4). Mortality was significantly lower for those with retinopathy regardless of duration of diabetes. This may be related to the use of statin therapy and hypertensive medication. Both of these class of medications had a significantly higher uptake in those with retinopathy (statin therapy: $78 \%$ vs 69\% (OR=1.55) and anti-hypertensive medication: $55 \%$ vs $47 \%(\mathrm{OR}=1.40)$ ).

\section{Survival analysis (type 2 diabetes only)}

In the Cox regression model including only type 2 diabetes there were 15852 patients. Mostly due to missing lipid level data, 792 (4.8\%) were excluded. There were 1856 (11.2\%) deaths among the included cases. Table 5 gives the effect size (HR) and the significance (p) for the variables in the final model. Only significant interactions are included.

As with the previous model, increasing age, being male, deprivation, smoking, and IHD all increased mortality risk. Duration of diabetes 5-10 years also increased mortality risk. Mortality risk was not increased with the longer duration $>10$ years. Hypertension now significantly affected mortality risk although the effect size was small. This confirmed the results in table 1 where there was a small increase in mortality for hypertensive type 2 diabetes patients compared with normotensive type 2 diabetes patients.

Being on statin therapy had a beneficial effect. Abnormal lipid levels whilst not on a statin significantly increased mortality risk for raised cholesterol and low HDL-cholesterol but not for triglycerides. Patients with foot ulcer/neuropathy had increased mortality risk but patients with retinopathy had reduced mortality risk (Table 5).

There were no significant interactions with duration of type 2 diabetes except for BMI at risk. Those with diabetes $<5$ years and BMI $>30 \mathrm{~kg} / \mathrm{m}^{2}$ had a lower mortality risk than those with diabetes duration of $>5$ years. This is in line with the results in table 3 . Age corrected in this model had $\mathrm{HR}$ for $\mathrm{BMI}>30 \mathrm{Kg} / \mathrm{m}^{2}$ at 0.58 for type 2 diabetes $<5$ years; at 0.78 for diabetes $5-10$ years and 0.81 for diabetes $>10$ years.

\section{Discussion}

The present "real world" study confirmed that patients with 
type 2 diabetes still have an increased mortality risk [3]. This large observational study had an accurate data base and is much larger than previous studies. Although some of the findings are not new, they are consistent with previous work and the size of the study allows us to rank the importance of the different risk factors. Increased mortality risk in type 2 diabetes was associated with being male while we previously demonstrated that the increased mortality risk in type 1 diabetes was associated with women [10]. Increased mortality was also associated with social deprivation, smoking, ischaemic heart disease and hypertension but not glycaemic control. Obesity and statin therapy appeared to be associated with reduced mortality risk. The present study showed that a male type 2 diabetes subject of 5-10 years duration who smoked, had hypertension and IHD plus lived in the most deprived area had a HR of 6.2 compared with a non-smoking, normotensive, non-diabetes subject without IHD living in the least deprived area.

The effect of duration on mortality risk in type 2 diabetes was interesting. The HRs for type 2 diabetes with duration less than 5 years and for 5-10 years were increased but fell slightly with duration greater than 10 years. This would appear to reflect selection bias among those with longer diabetes duration. These patients will have had diabetes for varying periods prior to the study and so there will have been mortalities in this group prior to the study period. Mortality risk among this "healthier" group of patients' will have underestimated the impact of duration and is an issue in any cross-sectional cohort study.

Social deprivation, measured by SIMD, which provides an areabased measure of deprivation, has previously been shown to be associated with type 2 diabetes and poorer outcome [14,15]. Although SIMD data zone may underestimate socioeconomic disparities, it is a relatively robust measure compared with previous indices used to measure social deprivation [11]. Debate continues as to the why social deprivation-related health inequalities are observed in many disease processes and populations $[14,16]$. In our study there was, however, no interaction seen between type 2 diabetes and social deprivation [14].

Numerous studies have demonstrated that good glycaemic control improves microvascular outcomes [3-6]. The relationship between good glycaemic control in type 2 diabetes and improvement in outcome has been more difficult to demonstrate $[5,6]$. Our study did not demonstrate better outcome in people with type 2 with a lower mean HbA1c. It may be that at this level of mean glycaemic control, differences in outcome are difficult to demonstrate and that five years is too short a period to demonstrate improvement in outcome. Furthermore, those patients with diabetes related or unrelated end stage disease may have had a lower HbAlc which would have skewed the results. It is also relevant to mention that The American BRAVO risk engine published in 2018 found a glycosylated haemoglobin level of $7.12 \%$, which was very close to the mean $\mathrm{HbAlc}$ in our study, was associated with the lowest risk for all-cause mortality in type 2 diabetes [17].

The prevalence of obesity in the type 2 diabetes patients in this study was greater than in the background population [15]. The presence of obesity appeared to protect against mortality risk in people with type 2 diabetes. With duration of diabetes, the presence of obesity in type 2 diabetes increased the mortality risk but the HR but still appeared protective. Previously, this effect has been described as the "Obesity Paradox" and has been demonstrated in type 1 diabetes [10], patients with coronary heart disease undergoing percutaneous coronary intervention, patients with hypertension, coronary heart disease and chronic heart failure [18-22]. Numerous mechanisms have been suggested and discussed elsewhere in detail [10,18-22].
Both hypertension and IHD were more prevalent in type 2 diabetes and became more prevalent with increased duration of diabetes. The mortality risk for hypertension and IHD was significantly higher for both normal and type diabetes subjects. When age was adjusted for in the survival model there was an interaction between hypertension and type 2 diabetes with little increase in mortality risk. This does not suggest that having hypertension is a positive factor for type 2 diabetes. It demonstrates that in type 2 diabetes, hypertension does not further increase your mortality risk significantly. It is likely that the mechanism(s) underlying hypertension and type 2 diabetes are similar $[23,24]$. In addition, hypertension may be better managed among type 2 patients with greater use of cardio-protective antihypertensive medications such as angiotensin converting enzyme inhibitors or angiotensin receptor blockers [25]. Furthermore, it is likely that hypertensive type 2 patients are more likely to be taking statin therapy than their non-diabetes counterparts [24].

This study also confirmed that those type 2 patients on statin therapy had a significantly lower mortality risk [26,27]. Patients not on statin therapy and with a raised total cholesterol or low HDL-cholesterol had a higher mortality risk than those with normal levels. For non-fasting triglycerides, mortality was lower for those with levels $>1.7 \mathrm{mmol} / \mathrm{L}$. An inverse association or lack of any association between triglycerides levels and outcome has been reported before [28]. Low triglyceride levels may result from malnourishment and weight loss related to chronic illness and the explanation may also be linked to the "Obesity Paradox" already discussed.

The relatively high level of statin therapy and treatment of hypertension probably reflects the QOF-driven [29] general improvement in diabetes care in Scotland and the UK. The management of type 2 diabetes is guideline-driven [30] and as a result management is very similar Scotland and UK-wide. This study also demonstrates that a significant portion of type 2 diabetes patients are not on appropriate anti-hypertensive and statin therapy leaving significant room for improvement [31]. We believe that our results are generalisable nationally.

\section{Conclusion}

This study confirmed that the all-cause mortality risk in people with type 2 diabetes remains elevated. In excess of $98 \%$ of our subjects were Caucasian so further work is required in other ethnic groups. Further work is also required to understand the interaction between hypertension and type 2 diabetes, why some patients of longer duration appear to be "protected", the role of the "Obesity Paradox" and why raised non-fasting triglycerides appears to reduce mortality risk.

\section{Declarations}

\section{Ethics approval and consent to participate}

Ethical opinion was not required for this study. The audit was registered with the Clinical Governance Department, NHS Ayrshire and Arran, UK and Caldicott Guardian approval was obtained from the Medical Director each General Practice.

\section{Consent for publication}

All data were anonymised.

\section{Availability of data and materials}

We are willing for all data to be made available.

\section{Competing interests}

There were no competing interests. 


\section{Funding}

The data collection and statistical analysis was supported by an Educational Grant from Astra Zeneca.

\section{Authors' contributions}

AC, MH and LC set up the study. AC, SG and NW undertook the literature search. LC collected the data while $\mathrm{MH}$ undertook the statistical analysis. All the authors, AC, MH, LC, SG, JB, MW and NW were involved in the writing of the paper.

\section{Acknowledgements}

N/A

\section{Authors' information}

N/A

\section{References}

1. Zimmet PZ, Magliano DJ, Herman WH, Shaw JE (2014) Diabetes: a $21^{\text {st }}$ century challenge. Lancet Diabetes Endocrinol 2: 56-64.

2. Gregg EW, Sattar N, Ali MK (2016) The changing face of diabetes complications. Lancet Diabetes Endocrinol 4: 537-547.

3. Collier A, Meney C, Hair M, Cameron L, Boyle JG (2020) Cancer has overtaken cardiovascular disease as the commonest cause of death in Scottish type 2 diabetes patients: A population-based study (The Ayrshire Diabetes Follow-up Cohort study). J Diabetes Investig 11: 55-61.

4. Gaede P, Lund-Andersen H, Parving H-H, Pedersen O (2008) Effect of a multifactorial intervention on mortality in type 2 diabetes. $\mathrm{N}$ Engl J Med 358: 580-591.

5. The UK Prospective Diabetes Study (UKPDS) Group (1998) Intensive blood-glucose control with sulphonylureas or insulin compared with conventional treatment and risk of complications in patients with type 2 diabetes (UKPDS 33). UK Prospective Diabetes Study (UKPDS) Group. Lancet 352: 837-853.

6. Holman RR, Paul SK, Bethel MA, Matthews DR, Neil HAW (2008) 10year follow-up of intensive glucose control in type 2 diabetes. N Engl J Med 359: 1577-1589.

7. Cholesterol Treatment Trialists' (CTT) Collaborators, Kearney PM, Blackwell L, Collins R, Keech A, et al. (2008) Efficacy of cholesterollowering therapy in 18,686 people with diabetes in 14 randomised trials of statins: a meta-analysis. Lancet 371: 117-125.

8. Afghahi $H$, Svensson MK, Pirouzifard M, Eliasson B, Svensson AM (2015) Blood pressure level and risk of major cardiovascular events and all-cause mortality in patients with type 2 diabetes and renal impairment: an observational study from the Swedish National Diabetes Register. Diabetologia 58: 1203-1211.

9. Mohiuddin SM, Mooss AN, Hunter CB, Grollmes TL, Cloutier DA, et al. (2007) Intensive smoking cessation intervention reduces mortality in high-risk smokers with cardiovascular disease. Chest 131: 446-452.

10. Collier A, Connelly PJ, Hair M, Cameron L, Ghosh S, et al. (2018) Mortality risk remains higher in individuals with type 1 diabetes: A population-based cohort study (the Ayrshire diabetes follow-up cohort [ADOC]). Diabetes Obes Metab 20: 1965-1971.

11. The Scottish Government (Riaghaltus na h-Alba) (2012) Statistics: Scottish Index of multiple deprivation 2012-Executive Summary.
12. Health and Social Care Integration Directorate (2013) 2013/14 General Medical Services Contract: Scottish Quality and Outcomes Framework Guidance. NHS Circular: PCA(M)2013)2.

13. Collier A, Ghosh S, Hair M, Waugh N (2015) Gender differences and patterns of cardiovascular risk factors in Type 1 and Type 2 diabetes: a population-based analysis from a Scottish region. Diabet Med 32: $42-46$.

14. Walker J, Colhoun H, Livingstone $\mathrm{S}$, McCrimmon R, Petrie J, et al. (2018) Type 2 diabetes, socioeconomic status and life expectancy in Scotland (2012-2014): a population-based observational study. Diabetologia 61: 108-116.

15. Collier A, Ghosh S, Hair M, Waugh N (2015) Impact of socioeconomic status and gender on glycaemic control, cardiovascular risk factors and diabetes complications in type 1 and 2 diabetes: a population based analysis from a Scottish region. Diabetes Metab 41: 145-151.

16. Frank J, Haw S (2011) Best practice guidelines for monitoring socioeconomic inequalities in health status: lessons from Scotland. Milbank Q 89: 658-693.

17. Shao H, Fonseca V, Stoecker C, Liu S, Shi L (2018) Novel Risk Engine for Diabetes Progression and Mortality in USA: Building, Relating, Assessing, and Validating Outcomes (BRAVO). Pharmacoeconomics 36: 1125-1134.

18. Romero-Corral A, Montori VM, Somers VK, Korinek J, Thomas RJ, et al. (2006) Association of bodyweight with total mortality and with cardiovascular events in coronary artery disease: a systematic review of cohort studies. Lancet 368: 666-678.

19. Costanzo P, Cleland JGF, Pellicori P, Clark AL, Hepburn D, et al. (2015) The obesity paradox in type 2 diabetes mellitus: relationship of body mass index to prognosis: a cohort study. Ann Intern Med 169: 610618.

20. Preston SH, Stokes A (2014) Obesity paradox. Obesity paradox: conditioning on disease enhances biases in estimating the mortality risks of obesity. Epidemiology 3: 454-461.

21. Bhaskaran K, Dos-Santaos-Silva I, Leon DA, Douglas IJ, Smeeth L (2018) Association of BMI with overall and cause-specific mortality: a population-based cohort study of 3.6 million adults in the UK. Lancet Diabetes Endocrinol 6: 944-953.

22. Chiolero A, Faeh D, Paccaud F, Cornuz J (2008) Consequences of smoking for body weight, body fat distribution, and insulin resistance. Am J Clin Nutr 87: 801-809.

23. de Boer IH, Bangalore $S$, Benetos $A$, Davis AM, Michos ED, et al. (2017) Diabetes and Hypertension: A Position Statement by the American Diabetes Association. Diabetes Care 40: 1273-1284.

24. Alberti KGMM, Zimmet $P$, Shaw J, IDF Epidemiology Task Force Consensus Group (2005) The metabolic syndrome-a new worldwide definition. Lancet 366: 1059-1062.

25. Olesen KKW, Madsen M, Egholm G, Thim T, Jensen LO, et al. (2017) Patients with Diabetes without Significant Angiographic Coronary Artery Disease have the Same Risk of Myocardial Infarction as Patients without Diabetes in a Real-World Population Receiving Appropriate Prophylactic Treatment. Diabetes Care 40: 1103-1110.

26. Pyŏrälä K, Pedersen TR, Kjekshus J, Faergeman O, Olsson AG, et al. (1997) Cholesterol lowering with simvastatin improves prognosis of diabetic patients with coronary heart disease. A subgroup analysis of the Scandinavian Simvastatin Survival Study (4S). Diabetes Care 20: 614-620. 
27. Colhoun HM, Betteridge DJ, Durrington PN, Hitman GA, Neil HAW, et al. (2004) Primary prevention of cardiovascular disease with atorvastatin in type 2 diabetes in the Collaborative Atorvastatin Diabetes Study (CARDS): multicentre randomised placebocontrolled trial. Lancet 364: 685-96.

28. Afsharian S, Akbarpour S, Abdi H, Sheikholeslami F, Moeini AS, et al. (2016) Risk factors for cardiovascular disease and mortality events in adults with type 2 diabetes-a 10-year follow-up: Tehran Lipid and Glucose Study. Diabetes Metab Res Rev 32: 596-606.

29. British Medical Association, National Health Service Employers (2009) Quality and Outcomes Framework guidance for GMS contract 2009/10.
30. SIGN (2017) Pharmacological management of glycaemic control in people with type 2 diabetes: SIGN 116 and 154. Health Improvement Scotland.

31. McGurnaghan S, Blackbourn LAK, Mocevic E, Panton UH, McCrimmon RJ, et al. (2019) Cardiovascular disease prevalence and risk factor prevalence in Type 2 diabetes: a contemporary analysis. Diabet Med 36: 718-725. 